\title{
Crenças de vulnerabilidade ao HIV/AIDS em adultos jovens em contexto universitário
}

\author{
Beliefs of vulnerability to HIV/AIDS in young adults in a university context \\ Creencias de vulnerabilidad al VIH/SIDA en adultos jóvenes en un contexto universitario
}

Recebido: 29/11/2021 | Revisado: 06/12/2021 | Aceito: 11/12/2021 | Publicado: 19/12/2021

\begin{abstract}
Aline Arruda Rodrigues da Fonseca
ORCID: https://orcid.org/0000-0002-5805-2650 Centro Universitário UNIESP, Brasil E-mail: aline.arruda@gmail.com

Luís Augusto de Carvalho Mendes ORCID: https://orcid.org/0000-0003-3841-9870 Centro Universitário UNIESP, Brasil

E-mail: luisaugustomendes@gmail.com

Márcio de Lima Coutinho

ORCID: https://orcid.org/0000-0003-1092-7566 Centro Universitário UNIESP, Brasil E-mail: marcio@iesp.edu.br

Solange Franci Raimundo Yaegashi ORCID: https://orcid.org/0000-0002-7666-7253

Universidade Estadual de Maringá, Brasil E-mail: solangefry@gmail.com

Fabrycianne Gonçalves Costa ORCID: https://orcid.org/0000-0003-2903-7555 Centro Universitário UNIESP, Brasil E-mail: prof1588@iesp.edu.br

Jaqueline Gomes Cavalcanti Sá

ORCID: https://orcid.org/0000-0002-3068-404X Centro Universitário UNIESP, Brasil E-mail: gomes.jaqueline@gmail.com
\end{abstract}

\begin{abstract}
Resumo
A vulnerabilidade é entendida como a possibilidade das pessoas adoecerem devido a condições diversas que são influenciadas pelo contexto em que elas estão inseridas. Neste sentido, tem como objetivo analisar as crenças e práticas preventivas de adultos jovens em contexto universitário acerca da Aids enquanto fatores que podem predispor à vulnerabilidade. Tratou-se de um estudo de campo, transversal e descritivo, adotando uma abordagem quantiqualitativa, realizado por meio de uma pesquisa de levantamento. A pesquisa foi realizada nos campi de Universidades públicas, Centros Universitários e Faculdades particulares do Estado da Paraíba. A amostra foi do tipo intencional e não-probabilística, constituída por 500 estudantes universitários, com idade entre 20 e 29 anos, distribuídos em diversos períodos letivos. Buscando saber se nas práticas do cotidiano, nas dinâmicas relacionais, e com mais acesso à informação há maior prevenção, buscando uma modificação nos cenários das interações sociais. $\mathrm{O}$ instrumento utilizado foi um questionário estruturado e autoaplicável. Para a análise textual computadorizada, os textos foram padronizados dentro dos critérios do freeware Iramuteq. Por meio da análise da Classificação Hierárquica Descendente foram encontradas cinco categorias temáticas: Invulnerabilidade, Impulsividade, Falta de Informação, Imaturidade Sexual e Sem Preservativo, que explicam as crenças relacionadas as práticas que apresentam vulnerabilidades sexuais e riscos de contaminação com HIV/AIDS. Assim, defende-se a adoção de políticas que visem o comportamento preventivo a partir de uma construção social, para além da vontade individual, mas que os elementos sociais e programáticos possam estar interligados, para a garantia da informação, preservativos e práticas baseadas no sexo seguro.
\end{abstract}

Palavras-chave: Crenças; Vulnerabilidade; HIV; AIDS; Estudantes universitários.

\begin{abstract}
Vulnerability is understood as the possibility of people becoming ill due to different conditions that are influenced by the context in which they are inserted. In this sense, it aims to analyze the beliefs and preventive practices of young adults in a university context about AIDS as factors that can predispose to vulnerability. It was a field, cross-sectional and descriptive study, adopting a quanti-qualitative approach, carried out through a survey. The research was carried out on the campuses of public universities and university centers and private colleges in the state of Paraíba. The sample was intentional and non-probabilistic, consisting of 500 university students, aged between 20 and 29 years,
\end{abstract}


distributed over different academic periods. Seeking to know whether in everyday practices, in relational dynamics, and with more access to information, there is greater prevention, seeking a change in the scenarios of social interactions. The instrument used was a structured and self-administered questionnaire. For the computerized textual analysis, the texts were standardized within the Iramuteq freeware criteria. Through the analysis of the Descending Hierarchical Classification, five thematic categories were found: Invulnerability, Impulsiveness, Lack of Information, Sexual Immaturity and No Condom, which explain the beliefs related to practices that present sexual vulnerabilities and risks of contamination with HIV/AIDS. Thus, it is defended the adoption of policies that aim at preventive behavior based on a social construction, beyond the individual will, but that social and programmatic elements can be interconnected, to guarantee information, condoms and sex-based practice safe.

Keywords: Beliefs; Vulnerability; HIV; AIDS; College students.

\section{Resumen}

La vulnerabilidad se entiende como la posibilidad de que las personas se enfermen por diferentes condiciones que están influenciadas por el contexto en el que se encuentran insertadas. En este sentido, tiene como objetivo analizar las creencias y prácticas preventivas de los jóvenes adultos en un contexto universitario sobre el SIDA como factores que pueden predisponer a la vulnerabilidad. Se trata de un estudio de campo, transversal y descriptivo, con enfoque cuanti-cualitativo, realizado a través de una encuesta. La investigación se llevó a cabo en los campus de universidades públicas y centros universitarios y colegios privados del estado de Paraíba. La muestra fue intencionada y no probabilística, compuesta por 500 estudiantes universitarios, con edades comprendidas entre 20 y 29 años, distribuidos en diferentes períodos académicos. Buscando saber si en las prácticas cotidianas, en las dinámicas relacionales, y con mayor acceso a la información, existe una mayor prevención, buscando un cambio en los escenarios de las interacciones sociales. El instrumento utilizado fue un cuestionario estructurado y autoadministrado. Para el análisis textual computarizado, los textos fueron estandarizados dentro de los criterios del freeware Iramuteq. A través del análisis de la Clasificación Jerárquica Descendente, se encontraron cinco categorías temáticas: Invulnerabilidad, Impulsividad, Falta de Información, Inmadurez Sexual y No Condón, que explican las creencias relacionadas con prácticas que presentan vulnerabilidades sexuales y riesgos de contaminación con VIH / SIDA. Así, se defiende la adopción de políticas que apunten a conductas preventivas basadas en una construcción social, más allá de la voluntad individual, pero que los elementos sociales y programáticos puedan estar interconectados, para garantizar información, condones y prácticas basadas en el sexo seguro.

Palabras clave: Creencias; Vulnerabilidad; VIH. SIDA; Estudiantes universitarios.

\section{Introdução}

Esta pesquisa tomou como ponto de partida o interesse em saber como os jovens se comportam frente à possibilidade de contrair o Vírus da Imunodeficiência Humana - HIV. Nesse sentido, buscou-se analisar as crenças e comportamentos diante da contaminação ao HIV/AIDS em estudantes universitários, público com boas condições de acesso a informações, por estarem inseridos no ensino superior, arena de conhecimento e trocas de informações científicas.

A justificativa deste estudo consiste no fato de que não apenas populações com um nível de escolaridade baixo ou menos favorecido economicamente têm se contaminado. Este artigo vem mostrar que também populações com escolaridade superior e melhor renda econômica, com melhores possibilidades de acesso a informações preventivas têm ainda crenças conservadoras sobre as formas de contágio e prevenção, podendo levá-los a situações de vulnerabilidade ao vírus da AIDS.

Em pesquisas com adolescentes foi constatado que eles tinham conhecimento sobre a transmissão do HIV (Garcia et al, 2021; Massena et al, 2021). Com base nessa informação buscou-se investigar o porquê de os jovens de hoje continuarem tendo comportamentos que os colocam em situação de vulnerabilidade. Esta sendo entendida como o "movimento de considerar a chance de exposição das pessoas ao adoecimento como resultante de um conjunto de aspectos não apenas individuais, mas também coletivos e contextuais que estão implicados com a maior suscetibilidade ao adoecimento e, concomitantemente, com a maior ou menor disponibilidade de recursos de proteção" (Ayres, França J., Calazans \& Salletti Filho, 2003, p. 123). Considerando-se, para estas explicações o estudo das crenças de Krüger (2011), entendidas como qualquer afirmativa feita pelo indivíduo, baseada em sua experiência pessoal, que pode ser interna ou externa, sendo apreendida dessa forma como representações mentais de processos cognitivos, afetivos, motivacionais e conotativos. 
O desenvolvimento adulto é extremamente diversificado, embora pareça caracterizar-se por duas necessidades básicas: a intimidade, alcançada por meio das amizades e dos relacionamentos amorosos e a produtividade, que geralmente se obtém por meio de um trabalho ou aprofundamento nos estudos (Papalia \& Martorell, 2021). Para a autora, as amizades e as parcerias amorosas são duas fontes fundamentais de intimidade no início da idade adulta. Estes diversos relacionamentos íntimos têm em comum não só as necessidades psicológicas que eles suprem, mas também os comportamentos que exigem. Todos eles compreendendo uma progressão, desde a atração inicial até a estreita conexão, cada papel exigindo uma doação de si mesmo, uma abertura e algum tipo de vulnerabilidade.

Para a compreensão dos significados sociais das juventudes modernas e contemporâneas, o essencial não é delimitar de antemão a faixa etária da sua vigência (Groppo \& Silveria, 2020). A faixa etária compreendida pela juventude não tem, segundo o autor, um caráter universal, ela torna-se resultado das necessidades e representações das sociedades sobre a sua própria dinâmica, sendo composta por um conjunto social que classifica indivíduos e normatiza seus comportamentos, definindo quais são seus direitos e deveres. Esta categoria configura-se no âmbito social, categorizando em grupos etários para uma melhor compreensão homogênea para simplificar e explicar os comportamentos sociais. Groppo e Silveria (2020) afirmam ainda que cada vez mais a juventude tem sido derivada da interpretação do momento cultural e social, sendo necessário, para compreendê-la, investigar aspectos sociodemográficos, como gênero, religião, condição econômica, etc.

E como seria este adulto jovem no contexto universitário? Nos dias atuais, muito se tem comentado sobre a educação de adultos. No universo acadêmico, existe um conjunto de fatores que afetam a forma como os estudantes buscam seu conhecimento e formação. Um primeiro fator a ser destacado, de acordo com Zabalza (2004) é que o ensino é superior, mas o estado de conhecimento ainda está em formação, pois o estudante é aprendiz. Outro ponto importante destacado pelo autor é o processo de maior acesso à educação superior, devido ao aumento no número de cursos, aumento no número de instituições de ensino superior e Fundo de Incentivo ao Ensino Superior, oferecido pelo Governo Federal, além da concentração maior de estudantes em determinados cursos, também foi assinalado por Osório (2021), o número de jovens que têm ingressado no ensino superior.

O presente artigo estuda o adulto jovem, inserido no contexto universitário por ser um grupo que nem sempre é contemplado no campo das políticas de saúde pública, inclusive por pertencerem a um grupo social a priori com mais recursos disponíveis para a saúde. As pesquisas com estudantes universitários homogeneízam os aspectos socioculturais, minimizando diferenças constatadas quando comparados com outros grupos, mesmo assumindo as diferenças existentes entre os indivíduos (Wagner, Maggi, Souza, C. \& Souza, R., 2010; Amorim, Abreu \& Coelho, 2021).

Deste modo Mann et al (1993) propuseram, na época, uma estrutura de análise que incorporava o comportamento individual, o âmbito coletivo e o social. O primeiro referia-se à autoavaliação, através de perguntas, que buscavam verificar o conhecimento, os comportamentos, o status social e o acesso à informação, avaliando como poderia ocorrer a transmissão da doença. A vulnerabilidade coletiva referindo-se à avaliação da capacidade estrutural e funcional dos programas de controle da epidemia e a vulnerabilidade social consistindo na avaliação das realidades sociais através de indicadores do Programa de Desenvolvimento das Nações Unidas.

Esse conceito de vulnerabilidade foi introduzido por Mann et al. (1993), por considerarem a multiplicidade de fatores envolvidos nas questões da AIDS e pelo fato de uma pessoa poder estar envolvida em alguma situação que possa torná-la vulnerável. Os autores acrescentaram ainda que as possibilidades de metabolizar informações e o poder de incorporá-las a mudanças práticas não dependeriam somente dos indivíduos, mas também de aspectos como acesso a meios de comunicação, grau de escolaridade, disponibilidade de recursos materiais, poder de influenciar decisões políticas, possibilidade de enfrentar barreiras culturais, etc. Todos esses aspectos deveriam ser, portanto, incorporados às análises de vulnerabilidade. 
A partir destas pressuposições, Ayres (2000) questionou sobre os determinantes que vão além do comportamento voluntário e individual das pessoas. Ele afirmou que passar informações é importante, pois a informação é um elemento fundamental para identificar quais os conjuntos de condições que efetivamente limitam as chances de as pessoas adotarem comportamentos mais protegidos.

Surge então outra perspectiva de vulnerabilidade proposta por Ayres (2000), na qual o modelo de vulnerabilidade aponta para o fato de que as pessoas se infectam pelo HIV não porque assumam individualmente comportamentos de risco, queiram correr riscos, ou desejem se expor ao vírus. É preciso entender o comportamento de um modo mais complexo, que tenha relação com o modo de vida das pessoas, como elas reproduzem suas crenças socialmente.

As vulnerabilidades apresentadas por jovens universitários ao HIV são diversas, e podem envolver situações como a precocidade da iniciação sexual, a necessidade de aceitação e inserção em grupos sociais, como um grupo que valorize a prática sexual, além do crescente consumo de álcool e outras drogas e questões de gênero (Wohlgemuth, Polejack \& Seidl, 2020). Além disso, há menor preocupação com a aquisição de infecções sexualmente transmissíveis (IST) do que com a prevenção da gravidez, mesmo quando os jovens estão bem informados, o que evidencia o caráter complexo da infecção pelo HIV a ser considerado durante as práticas de prevenção (Benitez \& Ponce, 2021), estes itens apontam para uma necessidade de se pensar a prevenção da gravidez, quando não planejada e às IST's, pois hoje, como assinalado por Pessoa, Vaz e Botassio (2021) tem-se um número bem maior de mulheres no ensino superior.

É importante, contudo, levar em consideração que os adultos jovens, estudantes universitários, possuem conhecimento sobre as formas de contaminação ao HIV/AIDS (Wohlgemuth, 2020). Este conhecimento é composto de informações adquiridas de forma voluntária e também involuntária, que transformam o modo como as pessoas entendem novos fatos e o conhecimento acumulado ao longo da vida, enquanto que as informações das particularidades da epidemia de AIDS é uma condição essencial para definir estratégias para lutar contra a sua progressão (Santos \& Oliveira, 2009).

No Brasil, estudo sobre a vulnerabilidade ao HIV em jovens universitários também foi realizado por Bezerra et al (2012), no qual foram verificados o não uso do preservativo em estudantes da área de saúde. Os motivos apontados pelos jovens foram diversos, tais como: o uso de anticoncepcional oral, confiança no parceiro, uso do preservativo ser desconfortável, falta do preservativo no momento da relação sexual e alergia ao látex do preservativo. Os resultados dessa pesquisa indicaram que os participantes não apresentaram o conhecimento suficiente sobre prevenção, o que foi apontado pelos autores com uma amostra de estudantes da área de saúde, na qual se almejava maior referência aos métodos de prevenção em geral.

Na vida cotidiana, a sexualidade é raramente pensada e vivida como atitudes e práticas coerentes com as opiniões e conhecimentos sobre a AIDS. Para Paiva (2012), a sexualidade é também elaborada nos termos de fatores e construtos descritos na literatura epidemiológica e da psicologia da saúde-como autoestima ou acesso à informação ou ainda sobre saber usar o preservativo. Para a autora há um critério que vai além destes pontos, ela refere-se ao momento do intercurso sexual e a todos os fatores nele envolvido, situação que a autora chamou de "cena", que é mais expressiva na dinâmica de fatores psicológicos e sociais, atualizada na atividade sexual.

\section{Metodologia}

Tratou-se de um estudo de campo, transversal e descritivo, adotando uma abordagem quanti-qualitativa, realizado por meio de uma pesquisa de levantamento. A pesquisa foi realizada nos campi de Universidades públicas e Centros Universitários e Faculdades particulares do Estado da Paraíba. 
A amostra foi do tipo intencional e não-probabilística, constituída por 500 estudantes universitários, com idade entre 20 e 29 anos, distribuídos em diversos períodos letivos. Para a inclusão dos participantes na amostra, foram adotados os seguintes critérios: I) Aceitar participar da amostra; II) Responder aos questionários de modo completo e; II) Ter idade de 20 anos a 29 anos.

Os participantes estavam distribuídos em 26 cursos de várias áreas, sendo $92 \%$ em instituições de ensino públicas (federais e estaduais). Grande parte $(73 \%)$ dos estudantes na Capital do Estado, apresentam média de idade de 22 anos $(\mathrm{DP}=$ 2,37), dos quais $82 \%$ situam-se na faixa etária de 20 à 24 anos, podendo ser considerados como adultos jovens. A maioria é do sexo feminino $(63 \%)$ e apenas $12 \%$ são casados.

A maioria se declara dependente financeiramente dos pais (56\%) ou do cônjuge (3\%), ou dependem do apoio institucional através de bolsa de estudos (7\%). Apenas 34\% afirmaram independência financeira com renda própria através de atividades profissionais. Independente da forma de manutenção, a renda familiar em geral, pode ser considerada média baixa, ou alcançando até a Classe C (IBGE, 2020).

A afiliação religiosa mais declarada foi a católica (57\%), seguido daqueles que afirmam não professar nenhuma religião. Em relação à religiosidade, $60 \%$ afirmaram alta ou média religiosidade. Estes dados são especificados na Tabela 1.

Tabela 1 - Perfil dos Participantes.

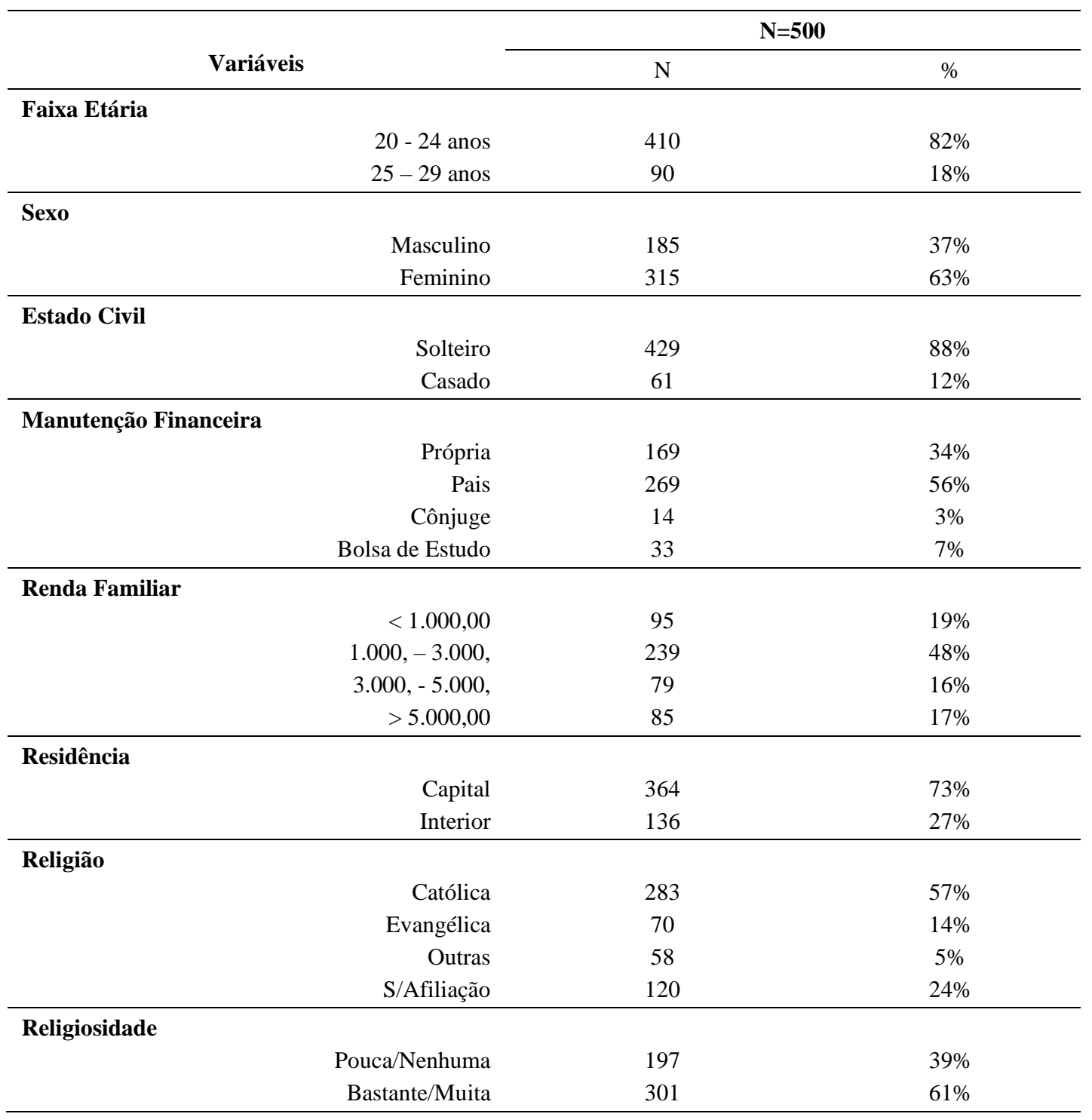


Research, Society and Development, v. 10, n. 16, e600101624045, 2021

(CC BY 4.0) | ISSN 2525-3409 | DOI: http://dx.doi.org/10.33448/rsd-v10i16.24045

\begin{tabular}{lrcc}
\hline Instituição de Ensino & Pública & 461 & \\
& Privada & 39 & $8 \%$ \\
\hline Tempo de Curso & & 347 & $69 \%$ \\
& $1^{\circ}$ ao $5^{\circ}$ período & 153 & $31 \%$ \\
\hline
\end{tabular}

Fonte: Autores.

O instrumento utilizado foi um questionário estruturado, autoaplicável, conforme a descrição a seguir: a) Questionário sociodemográfico com informações sobre idade, sexo, status conjugal, cidade de domicílio, renda familiar, religião, religiosidade, vínculo institucional, curso e período e; b) Explicações para Vulnerabilidade (Uma única questão aberta, dissertativa, respondida por escrito, questionando sobre os motivos da contaminação de jovens pelo HIV/AIDS).

Após a autorização dos gestores das instituições para realização da pesquisa, passou-se a etapa de aplicação do instrumento atendendo a disponibilidade dos professores em sala de aula.

O desenvolvimento do estudo seguiu os critérios que preconizam pesquisas com seres humanos conforme Resolução no 196/96 (Sobre Pesquisa Envolvendo Seres Humanos, Ministério da Saúde, Conselho Nacional de Saúde, 1996), registrado na Plataforma Brasil sob o registro CAAE 53233921.1.0000.5184. Este estudo faz parte de uma pesquisa maior que resultou em uma tese de doutorado.

Para a análise textual computadorizada, os textos foram padronizados dentro dos critérios do freeware Iramuteq. Devido a quantidade pequena de textos e as respostas escritas de forma reduzida, optou-se pelo agrupamento em 15 categorias temáticas identificadas de forma livre pelos pesquisadores. Assim, a entrada do corpus foi realizada por meio de 15 textos e as análises possibilitaram a realização de uma Classificação Hierárquica Descendente (CHD), que indica contextos lexicais por meio da co-ocorrência de palavras, e uma Análise de Similitude, que indica o grau de relacionamento entre os termos, de acordo com os critérios definidos pela literatura (Ratinaud, 2014; Camargo \& Justo, 2013)

\section{Resultados}

O corpus apresentou 2226 ocorrências com 644 palavras distintas. Após a análise lexical básica, o material foi submetido a uma Classificação Hierárquica Descendente (CHD), que desdobrou os 15 textos iniciais em 75 segmentos de texto, com uma média de frequência média de 30 formas por segmento. Para o Dendograma foram considerados 59 segmentos (79\%) do total inicial.

Para cada classe foram listadas as 08 palavras com maior capacidade explicativa, calculada por meio do $\mathrm{X}^{2}$ (Quiquadro), que indica o nível de associação significativa $(\mathrm{p}<0,01)$ de cada item com a classe na qual está inserida, como pode ser observado na Tabela 2, a seguir. 
Tabela 2 - CHD e Dendrograma (adaptado em forma de tabela).

\begin{tabular}{|c|c|c|c|c|c|c|c|c|c|c|c|}
\hline \multicolumn{12}{|c|}{ Corpus dos roteiros - 59 ST $(79 \%)$} \\
\hline \multicolumn{10}{|c|}{ Subcorpus A $(83 \%)$} & \multirow{2}{*}{\multicolumn{2}{|c|}{$\begin{array}{c}\text { Classe } 2 \text { (17\%) } \\
\text { Invulnerabilidade }\end{array}$}} \\
\hline \multirow{4}{*}{\multicolumn{2}{|c|}{$\begin{array}{l}\text { Classe } 6(18,6 \%) \\
\text { Impulsividade }\end{array}$}} & \multicolumn{8}{|c|}{ Subcorpus B $(64,4)$} & & \\
\hline & & \multicolumn{6}{|c|}{ Subcorpus C $(49,2 \%)$} & \multirow{2}{*}{\multicolumn{2}{|c|}{$\begin{array}{c}\text { Classe } 5(15,2 \%) \\
\text { Drogas }\end{array}$}} & & \\
\hline & & \multirow{2}{*}{\multicolumn{2}{|c|}{$\begin{array}{c}\text { Classe } 1(15,2 \%) \\
\text { Falta de } \\
\text { Informação }\end{array}$}} & \multicolumn{4}{|c|}{ Subcorpus D (34\%) } & & & & \\
\hline & & & & \multicolumn{2}{|c|}{$\begin{array}{c}\text { Classe } 3(20,3 \%) \\
\text { Imaturidade } \\
\text { Sexual }\end{array}$} & \multicolumn{2}{|c|}{$\begin{array}{l}\text { Classe } 4(13,7) \\
\text { Sem } \\
\text { Preservativo }\end{array}$} & & & \\
\hline$\overline{X^{2}}$ & Forma & $\mathbf{X}^{2}$ & Forma & $\mathbf{X}^{2}$ & Forma & $\mathbf{X}^{2}$ & Forma & $\mathbf{X}^{2}$ & Forma & $\mathbf{X}^{2}$ & Forma \\
\hline 40,4 & consequência & 27,9 & falta & 17,0 & sexual & 32,7 & camisa & 23,8 & seringa & 39,1 & acontecer \\
\hline 34,7 & agir & 17,7 & informação & 15,7 & vez & 12,9 & usar & 23,8 & droga & 38,9 & nunca \\
\hline 23,8 & impulso & 17,6 & diálogo & 11,7 & mais & 10,2 & mesmo & 23,8 & álcool & 26,8 & ruim \\
\hline 14,6 & momento & 13,7 & método & 7,9 & acabar & 10,1 & risco & 22,6 & uso & 22,2 & acreditar \\
\hline 13,6 & ato & 8,5 & campanha & 6,6 & relação & 8,6 & preservativo & 17,6 & aumentar & 15,4 & coisa \\
\hline 13,6 & deixar & 6,7 & jovem & 4,2 & vida & 8,6 & sexo & 13,7 & como & 10,3 & pensamento \\
\hline 11,9 & prazer & 4,0 & consciência & 4,2 & conhecer & 7,6 & torna-se & 6,5 & também & 8,5 & jovem \\
\hline 11,5 & pensar & 4,0 & conhecimento & 4,2 & querer & 2,5 & muito & 6,5 & além & 7,2 & só \\
\hline
\end{tabular}

Fonte: Análise textual pelo Iramuteq. Nota: $\mathrm{X}^{2}=$ valor do Qui-quadrado.

A primeira partição distinguiu o subcorpus que originou diretamente a classe 2 "Invulnerabilidade" do restante do material textual (subcorpus A). Numa segunda partição, o "subcorpus A" foi dividido em duas categorias: uma relativa a classe 6 "Impulsividade" e o subcorpus B. Na terceira partição, o "subcorpus B” desmembrou-se entre a classe 5 "Drogas" e o subcorpus Subcorpus C. Já o "Subcorpus C” se dividiu na Classe 1 "Falta de Informação" e no Subcorpus D. E, por fim, o “Subcorpus D” originou a Classe 3 "Imaturidade Sexual” e a Classe 4 "Sem preservativo".

A Classe 2 "Invulnerabilidade" explicou 17\% dos segmentos analisados e destacou os termos: acontecer, nunca, ruim, acreditar, coisa, pensamento, jovem e só. Nesta categoria estão as respostas associadas a ideia de que muitos jovens acham que não correm riscos, mesmo adotando práticas sexuais desprotegidas. Essa ideia ora exposta de invencibilidade e superproteção. As pessoas na faixa etária estudada podem, portanto, estar mais propensas aos riscos, devido às características comuns à idade de descobertas e mudanças de vida, como foi descrito pelos próprios jovens, há uma ideia de blindagem diante do perigo.

A Classe 6 "Impulsividade" explicou 18,6\% do corpus, com destaque para os termos: consequência, agir, impulso, momento, ato, deixar, prazer e pensar. Em conjunto, por meio das formas é possível observar a vulnerabilidade como componente mais voltado para fatores individuais, presente na fala dos jovens, ainda mesclado com as concepções que se tem, no senso comum, da adolescência. A ideia de agir por impulso, da busca pelo prazer sem a preocupação imediata de contrair alguma doença.

Outro fator relacionado às vulnerabilidades está descrito na Classe 5 "Drogas", que explicou 15,2\% dos textos. Os termos com maior relevância foram: seringa, droga, álcool, uso, aumentar, como, também e além. Embora não tenha sido interesse focal da investigação da presente pesquisa, foi um dado que emergiu nas falas dos participantes, como fator 
relacionado às vulnerabilidades que contribuem para a propagação do HIV/AIDS, já que, socialmente, o comportamento de consumir bebidas alcoólicas é visto como facilitador de interações sociais.

A "Falta de informação" (Classe 1, que explicou 15,2\% dos segmentos) destacou as formas: falta, informação, diálogo, método, campanha, jovem, consciência e conhecimento. Esses termos ressaltam o argumento da falta de orientação quanto ao HIV/AIDS. Assim, as campanhas sobre a AIDS e o acesso à informação são pontos sempre necessários para discussão e estão relacionados ao que Ayres et al. (2003) chamou de vulnerabilidade programática, na qual, os principais objetivos dos programas nacionais direcionados à prevenção, controle e assistência em HIV/AIDS são: proporcionar informações e educação de forma abrangente, sustentada e coerente; implementar ações preventivas; fomentar pesquisas e colaborar na aderência ao tratamento. As afirmativas descritas pelos jovens relacionadas a crenças conservadoras sobre a epidemia, o que demonstra ainda desinformação e como pode ser melhor explicado nas respostas abaixo apresentadas.

A Classe 3 "Imaturidade Sexual", que explicou 20,3\% do texto analisado e apresentou os termos: sexual, vez, mais, acabar, relação, vida, conhecer e querer. Os textos que compuseram esta classe podem estar relacionados a idade dos participantes e características da iniciação sexual ou aos mitos relacionados ao amor romântico, que levam em consideração a confiança entre parceiros, porém diminuem as relações baseadas em ações de relações sexuais seguras.

Por fim, a Classe 4 "Sem Preservativo", explicando 13,7\% dos segmentos de textos, destacou as formas: camisa, usar, mesmo, risco, preservativo, sexo, torna-se e muito. Neste contexto, o uso do preservativo é visto de forma negativa, sendo o insumo associado ao desconforto, apontado por muitos jovens e está diretamente relacionado a uma crença construída socialmente de que o preservativo diminui o prazer, incomoda, é desinteressante, dentre outras crenças, nas quais algumas respostas foram dadas como uma condição na qual o prazer não pode estar vinculado ao uso do preservativo.

Por meio do Iramuteq foi possível realizar uma Análise de Similitude, que apresentou as relações entre as palavras. Assim, é possível ver uma árvore de palavras com as ramificações indicando a força das relações entre os termos, como pode ser detalhado na Figura 1. Para uma melhor visualização do resultado, as relações entre as palavras foram submetidas ao Freeware Gephi.

A palavra central da Análise de Similitude foi o termo "não", indicando que as respostas dos entrevistados apresentaram maiores conexões com a não realização dos cuidados necessários para a proteção. No agrupamento central também apareceram as palavras "pensar" e "mesmo" com forte relação ao "não" indicando que as ações são impensadas. Por sua vez, a forma "pensar" relacionou-se fortemente com "prazer", o que indica que os jovens pensam no prazer "momentâneo". Os demais agrupamentos circunvizinhos apresentaram conexões centralizadas pelas palavras "muito", "jovens", "achar", "uso", "sexual”, "vez", "porque” e "falta". Os detalhes das relações entre as palavras podem ser visualizadas em detalhe na Figura 1, a seguir. 
Figura 1. Análise de Similitude.

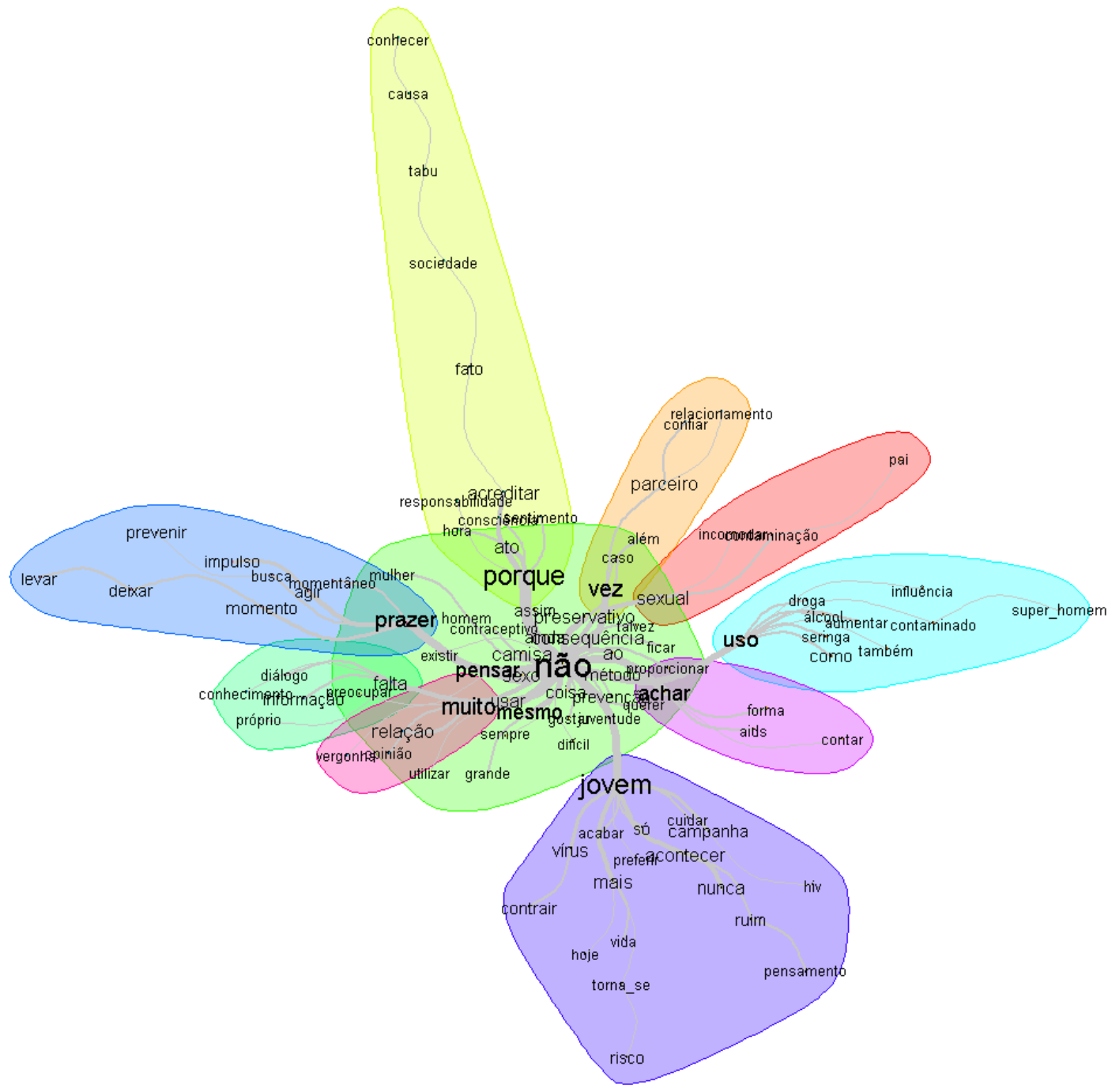

Fonte: Autores, por meio do Iramuteq.

\section{Discussão}

Em um primeiro momento é possível observar a vulnerabilidade como componente mais voltado para fatores individuais, presente na fala dos jovens, ainda mesclado com as concepções que se tem, no senso comum, da adolescência. A ideia de agir por impulso (impulsividade), da busca pelo prazer sem a preocupação imediata de contrair alguma doença, como pode-se observar no trecho a seguir: "Porque ele quer prazer intenso e muitas vezes faz as coisas por impulso" (Feminino, 24 anos)

A percepção Invulnerabilidade, na qual foram comuns respostas associadas a ideia de que muitos jovens acham que não correm riscos, mesmo adotando práticas sexuais desprotegidas, como este exemplo de fala: “O ser humano tem uma cultura de acreditar que nada de ruim vai atingi-lo" (Feminino, 26 anos)

A invulnerabilidade e irresponsabilidade é então resultante de uma imaturidade, assim descrita pelos próprios participantes. Esta imaturidade gera comportamentos irresponsáveis, levando à percepção de invulnerabilidade e põe em 
questão o acesso a informações preventivas a decisão de assumir comportamentos preventivos ou de exposição ao HIV. Assim, além dessas crenças, existe outro motivo apontado pelos jovens, como é descrito na fala a seguir: "Falta de reflexão de cuidado com a própria saúde e a saúde do parceiro" (Masculino, 22 anos).

A imaturidade foi apontada pelos jovens como outro fator de risco que expõe os jovens a contrair o HIV/AIDS. Semelhante à categoria de invulnerabilidade e impulsividade na qual a busca pelo prazer, tão comum aos jovens, vem associada a pouca preocupação diante da contaminação.

Outra palavra que emergiu nas falas foi drogas, apontado pelos jovens, embora não seja do interesse de investigação da presente pesquisa, foi um dado que emergiu nas falas dos participantes, como apresentado a seguir: "Levando em conta também o alto índice de uso de drogas, que faz com que aumente ainda mais" (Masculino, 20 anos).

O consumo de álcool associado à desinibição não esteve presente no questionário, mas emergiu nas "falas" dos participantes apontando para novas possibilidades de pesquisa, já que, socialmente, o comportamento de consumir bebidas alcoólicas é visto como facilitador de interações sociais.

Estas falas mostram como existem crenças que podem levar a um maior risco à vulnerabilidade ao HIV, pois emergem muito mais que fatores apenas de conhecimento, na qual estão envolvidas as relações de poder e confiança quanto ao uso do preservativo, assim como nos resultados da Escala de Crenças acerca do uso do preservativo são direcionados para o não uso quando se conhece e confia no (a) parceiro (a).

Diante deste contexto, o uso do preservativo é visto de forma negativa, na subcategoria gênero, sendo o insumo associado ao desconforto, apontado por muitos jovens e está diretamente relacionado a uma crença construída socialmente de que o preservativo diminui o prazer, incomoda, é desinteressante, dentre outras crenças, nas quais algumas respostas foram dadas como uma condição na qual o prazer não pode estar vinculado ao não uso do preservativo, conforme pode ser observado na descrição seguinte: "Pois o uso do preservativo atrapalha e muito a relação sexual" (Masculino, 21 anos).

A falta de informação aponta para como estão sendo divulgadas e absorvidas as campanhas sobre a AIDS e o acesso à informação sendo pontos sempre necessários para discussão e estão relacionados ao que Ayres et al. (2003) chamou de vulnerabilidade programática, na qual, os principais objetivos dos programas nacionais direcionados à prevenção, controle e assistência em HIV/AIDS são: proporcionar informações e educação de forma abrangente, sustentada e coerente; implementar ações preventivas; fomentar pesquisas e colaborar na aderência ao tratamento. As afirmativas descritas pelos jovens relacionadas a crenças conservadoras sobre a epidemia esteve em $20 \%$, o que demonstra ainda desinformação e como pode ser apresentado na fala: "É necessário, mais campanhas para o uso do preservativo, assim como se prevenir contra vírus do HIV" (Feminino, 20 anos).

Desse modo, diante de tantas divergências sobre o uso ou não uso do preservativo e as crenças envolvidas sobre o insumo, as questões também discrepantes apontadas sobre as campanhas preventivas e a construção das atitudes de cada jovem compartilhada no grupo sobre a prevenção, foi possível verificar que muitos jovens são vulneráveis a contrair o HIV, mas não se percebem dessa forma. As crenças acerca da AIDS influenciam diretamente forte sobre quais informações serão incorporadas cognitivamente para conduzir estes jovens ou distanciá-los de práticas preventivas.

A iniciação sexual dos jovens deste estudo ocorreu entre os 17 e os 20 anos. Esses resultados demonstram que a idade da iniciação sexual aumentou em relação a estudos anteriores (Ferreira et al, 2020) realizados com adolescente que apontavam para uma idade precoce de 14 anos, mas corrobora o mesmo estudo ao citar a idade do (a) parceiro (a) como sendo um pouco mais velho que o jovem.

O parceiro da primeira relação foi, geralmente, o namorado, principalmente para as mulheres, perpetuando-se a ideia do amor romântico, na confiança no parceiro para a primeira experiência sexual, conforme estudos anteriores realizados por Bielenki et al (2019), o que tem apontado ainda para a feminização da AIDS estar relacionada a esta concordância em não se 
solicitar o preservativo, já que há a "seguridade" de um relacionamento estável. Contudo, Panarra et al (2017) apontam para o fato de que perante a descoberta do diagnóstico de soropositividade para o HIV, a mulher vivencia sentimentos de dúvidas e insegurança, levando-as a vivenciar uma situação de crise. O diagnóstico de soropositividade ocasiona sempre um choque, ocasionando um trauma de natureza física, emocional e social.

Não obstante esta tendência, alguns resultados podem ser destacados, a exemplo da idade da iniciação sexual mais avançada, visto que em estudos anteriores neste mesmo Estado, com adolescentes, a iniciação sexual se dava, em média aos 14 anos (Ferreira et al, 2020). Destarte, a idade da primeira relação sexual (genital) aumentou. Para o sexo masculino, um pouco mais cedo que para o sexo feminino (16,5 anos diante de 18, 4 anos para as mulheres) e com parceiras com maior idade, geralmente tendo como parceira a namorada (26\%), parceira ocasional (ficante) (23\%) e amiga (22\%). Em estudo realizado com uma amostra de adolescentes, Silva et al. (2020) apontou para uma iniciação da prática sexual bem mais jovem do que os achados da presente tese, isso pode supor que os comportamentos relacionados à sexualidade de alguns jovens sejam recorrentes desde a fase da adolescência.

Outro ponto de destaque é o número de jovens que tem a primeira relação sexual com desconhecidos, ou seja, pessoas que conheceram naquele dia, ( $21 \%$ do sexo masculino), reforçando a crença de que um homem não deve recusar uma interação sexual (Guerriero, Ayres \& Hearst, 2002). Estes dados apontam para a ideia da masculinidade na qual o homem tem que estar sempre disposto a uma interação sexual, assim como tem que ser sexualmente ativo e ter várias parceiras para o grupo, portanto, qualquer oportunidade de prazer sexual não deve ser desperdiçada.

Para o sexo feminino geralmente o primeiro parceiro foi o namorado (81\%), também com idade mais avançada. Esses resultados implicam que o jovem virgem, de idade inferior ao seu parceiro de iniciação sexual não representa uma ameaça de contaminação com alguma IST, mas ao se relacionar sexualmente com uma pessoa mais velha, implica dizer que este jovem pode estar se relacionando com todos os outros parceiros anteriores de seu atual companheiros, os quais não fizeram uso de preservativo. Por outro lado, observa-se um número considerável de jovens que ainda não tiveram a primeira relação sexual (12\% para o masculino e $36 \%$ feminino).

Em relação à orientação sexual, a maioria declarou ser heterossexual (94\%). Portanto todas as discussões desta pesquisa estão relacionadas às questões sociais do masculino e do feminino e do quanto estas relações se estabelecem socialmente desde a negociação do uso do preservativo, seu manuseio e compra até mesmo a aquisição do insumo e as crenças construídas acerca da AIDS.

As práticas preventivas neste estudo referem-se ao uso do preservativo masculino. Sobre o método preventivo mais utilizado, o preservativo masculino (60\%), foi o mais assinalado, seguidos da pílula anticoncepcional (28\%). Aqui observa-se que a pílula anticoncepcional é muito utilizada como método preventivo, sendo este método entendido pelos jovens como necessário para evitar a gravidez, mas não apontam para a prevenção contra IST's, o que pode colocá-los em situação de vulnerabilidade, isso leva a um questionamento sobre o que eles têm percebido como prevenção necessária e novamente o prazer é colocado à frente da prevenção, prazer este que, no entendimento dos jovens, não pode ser atingido ao se usar o preservativo.

Os resultados apontaram para um uso inconstante do insumo, estando em consonância ao estudo de Guerra et al (2020), o qual afirma que o comportamento de não usar o preservativo é apontado pelos jovens a partir de uma questão estética, do medo de perder a ereção e na perda de sensibilidade no homem e na mulher, essas justificativas colocam os jovens normalmente em maiores situações de vulnerabilidade, pois a preocupação deles está no desempenho sexual e no prazer, muito mais do que na preocupação preventiva às IST's.

Ao se investigar a percepção de vulnerabilidade à AIDS, os dados apresentados trazem questionamentos sobre a construção das crenças acerca da AIDS nos jovens, pois existe uma inconstância no que eles julgam correto e nos 
comportamentos preventivos a exemplo de apenas $22 \%$ dos jovens terem feito a testagem para o HIV, diante de apenas $19 \%$ afirmarem continuar um relacionamento se descobrissem um diagnóstico soropositivo para o parceiro. Assim, como afirmou Krüger (2011), no processo de formação das crenças, é importante considerar o grau de aceitação subjetiva das mesmas, que vai influenciar diretamente nas tomadas de decisão, assim quando o jovem não se vê como vulnerável, o comportamento mais comum é o de não usar o preservativo, mas isso não serve para o outro, pois este pode ser visto como vulnerável, e esta percepção passa apenas por um julgamento cognitivo não se buscando a certeza do diagnóstico através da testagem.

De modo semelhante, a vulnerabilidade parece ocorrer devido às crenças individuais relacionadas a uma não percepção de poderem se contaminar. Assim, fica um questionamento sobre que crenças foram construídas para embasar esta percepção fazendo-se necessário retomar Krüger (2011), quando o autor aponta para a natureza ontológica das pessoas, na qual se encontram inter-relacionados de modo inseparável, processos, estados e conteúdos psicológicos, mas que ocorrem nas dinâmicas relacionais cotidianas.

Assim, para um aprofundamento nos resultados apresentados pelos jovens nos dados quantitativos, procedeu-se a análise dos dados qualitativos, ou seja, a análise da questão aberta. Sobre ela, através da classe temática Explicações de Vulnerabilidade e de suas três categorias que emergiram, foi possível compreender o que os jovens apontaram como motivos de não se estar fazendo uso do preservativo nas relações sexuais.

Nesse sentido, a junção dos fatores invulnerabilidade e irresponsabilidade justificam-se pela imaturidade descrita pelos jovens. Assim, como afirma Ayres et al. (2003), esses aspectos, mesmo tendo um caráter de ordem social, são introjetados pelo indivíduo de modo particular, individual. Um critério que corrobora esta subcategoria é a idade da iniciação sexual, que, embora tenha sido um pouco mais tardia (por volta dos 17 anos), em comparação a estudos recentes desenvolvidos com adolescentes (Silva et al., 2018) que apontava para uma idade de 14 anos, ainda assim pode estar indicando uma precocidade se for tomado como ponto de referência preventiva o uso do preservativo, por isso, neste momento é possível compreender quando os jovens descrevem o fator imaturidade como justificativa de comportamentos que possam levar à vulnerabilidade ao HIV, assim como a relação estabelecida entre o jovem e a pessoa do intercurso sexual, que para muitos jovens tratou-se de ser um parceiro ocasional. Outro fator continua sendo o uso irregular do preservativo, onde foi possível verificar que apenas $45 \%$ dos jovens portavam o insumo no momento do intercurso sexual e ainda que $32 \%$ destes jovens ainda sentem-se constrangidos em adquirir o preservativo.

A partir do exposto, verifica-se na perspectiva de Krüger (2011), uma crença conservadora relacionada à impulsividade, a ideia de que ser homem significa ter que correr riscos. Os jovens sabem de suas práticas sexuais desprotegidas, como perguntado no estudo, mas não são as informações - como a informação de ser importante usar o preservativo - que reforçam suas crenças de invulnerabilidade, portanto, não são comportamentos preventivos assumidos pelos jovens.

\section{Considerações Finais}

Estudar a vulnerabilidade à AIDS em jovens através das crenças construídas por esta população é uma possibilidade de conhecer que fatores podem levar a uma maior ou menor predisposição de exposição ao vírus. São as elaborações sociais construídas desde o início da epidemia, há trinta anos, que, embora tenha passado por significativas modificações, ainda tem apresentado crenças distorcidas sobre as formas de contaminação e sobre as pessoas que convivem com o HIV.

O modelo de vulnerabilidade ora apresentado busca fornecer elementos para avaliar objetivamente as diferentes chances que todo e qualquer indivíduo tem de se contaminar, dado o conjunto formado por certas características individuais e sociais de seu cotidiano, julgadas relevantes para a sua maior exposição ao HIV ou sua menor chance de proteção. 
Os resultados apontam para a necessidade não só da manutenção das pesquisas em torno da AIDS e do estudante universitário, como também de mudanças nas características de suas propostas. A manutenção desse trabalho e mesmo a sua expansão justifica-se em função do aumento das notificações de AIDS e do aumento notável no número de estudantes do ensino superior no país.

Assim, o comportamento preventivo deve partir de uma construção social, para além da vontade individual, mas que os elementos sociais e programáticos possam estar interligados, constituindo um único critério de prevenção, e que questões culturais, políticas, de gênero e faixa etária não sejam fatores que possam estigmatizar essas práticas preventivas. A diversidade de comportamentos sexuais e afetivos deve ser sempre entendida com suas singularidades contidas em cada relacionamento.

\section{Referências}

Amorim, L. O. A. B.; Abreu, M. A. G. M.; Coelho, M. T. A. D. (Orgs.) (2021). Saúde na educação superior: o que estudantes e professores têm a dizer? Salvador: EDUFBA. Modo de acesso: https://repositorio.ufba.br/ri/handle/ri/34206 ISBN: 978-65-5630-186-0 (Epub)

Ayres, J. C. R. M., França Junior, I., Calazanz, G. J. \& Saletti Filho, H. C. (2003). O conceito de vulnerabilidade e as práticas de saúde: Novas perspectivas e desafios. In: Ayres, J. R. C. M., Freitas, A. C., Santos, M. A. S., Saletti Filho, H. C. \& França Júnior, I. (2003). Adolescence and AIDS: evaluation of a preventive education experience among peers. Interface - Comunicação, Saúde, Educação, 7(12), p.113-28.

Ayres, J. R. C. M. (2000). Cidadania, vulnerabilidade e prevenção de HIV/AIDS. in Pinto, T.; Telles, I. S. (Orgs.). AIDS e escola: reflexões e propostas do EDUCAIDS. $2^{\text {a }}$ Ed. São Paulo: Cortez; Pernambuco: UNICEF.

Benitez, S. R. S, Ponce, M. A. Z. (2021). Conhecimento de universitários em relação à prevenção combinada e gestão de risco do HIV/AIDS. Anais do $12^{\circ}$ Fórum Científico UNIFUNEC: Educação, Ciência e Tecnologia, 29 de setembro a $1^{\circ}$ de outubro, Santa Fé do Sul (SP), 12 (12), ISSN: $2318-745 X$.

Bezerra, E. O., Chaves, A. C. P., Pereira, M. L. D. \& Melo, F. R. G. (2012). Análise da vulnerabilidade sexual de estudantes universitários ao HIV/AIDS. Revista da Rede de Enfermagem do Nordeste. 13(5), 1121-31.

Bielenki, C. R. Z., Schermann, L. B., Santos, A. M. P. V., Arossi, G. A. \& Béria, J. U. (2019). Sexualidade na adolescência em tempos de Aids: um estudo com escolares. Aletheia, 52(2), 135-146. http://pepsic.bvsalud.org/scielo.php?script=sci_arttext\&pid=S1413-03942019000200011\&lng=pt\&tlng=pt.

BRASIL. Ministério da Saúde. Conselho Nacional de Saúde. Resolução n. 196, de 10 de outubro de 1996. Aprova as diretrizes e normas regulamentadoras de pesquisas envolvendo seres humanos. Brasília, Diário Oficial da União, 16 out. 1996.

Camargo, B. V., Justo, A. M. (2013). IRAMUTEQ: um software gratuito para análise de dados textuais. Temas em Psicologia, 21(2), 513-518.

Ferreira, AP. C., Barreto, ACM, Couto, LL, Oliveira, LPM de, \& Teixeira, SVB (2020). Iniciação sexual na adolescente: narrativas de vida de mulheres. Research, Society and Development, 9 (10), e2859108336. https://doi.org/10.33448/rsd-v9i10.8336

Garcia, E. C.; Costa, I.R.; Oliveira, R.C.; Silva C. R. L.; Góis A. R. S.; Abrão, F. M. S. (2021). Representações sociais de adolescentes sobre a transmissão do HIV/AIDS nas relações sexuais: vulnerabilidades e riscos. Esc. Anna. Nery, 26. https://doi.org/10.1590/2177-9465-EAN-2021-0083

Groppo, L. A; Silveira, I. B. (2020). Juventude, classe social e política: reflexões teóricas inspiradas pelo movimento das ocupações estudantis no Brasil. Argum., Vitória, 12 ( 1), p. 7-21, jan./abr. ISSN 2176-9575.

Guerra, F., Otaviano, R., Ramos, R., Damião, M., \& Zanini, E. (2020). Comportamento Sexual de Estudantes Universitários: Um Estudo de Revisão. Fag Journal of Health (Fjh), 2(2), 300-306. https://doi.org/10.35984/fjh.v2i2.175

Guerriero, I., Ayres, J. R. C. M. \& Hearst, N. (2002). Masculinidade e vulnerabilidade ao HIV de homens heterossexuais, São Paulo, SP. Revista de Saúde Pública, 3(6), 50-60. www.fsp.usp.br/rsp

IBGE - Instituto Brasileiro de Geografia e Estatística. Apresentação - PNAD Contínua Rendimentos todas as fontes - 2020. Rio de Janeiro: IBGE, 2021. https://agenciadenoticias.ibge.gov.br/

Krüger, H. (2011). Ideologias, sistemas de crenças e atitudes. In: Torres, A. R. R. et al. (Orgs.). Psicologia Social: temas e teorias. Brasília: Technopolitik.

Mann, J., Tarantola, D. J. N. \& Netter, T. W. (1993). A AIDS no Mundo [AIDS in the World]. Organizadores da Edição Brasileira: Richard Parker, Jane Galvão, José Stalin Pedrosa. Rio de Janeiro: Relume-Dumará: ABIA: IMS/UERJ.

Massena, T. P., Lima, A. S. D., Soares, V. L., Ramos, I. S. M., Rezende, G. L. (2021). Aspectos da vulnerabilidade de adolescentes frente ao HIV. Revista Multidisciplinar Em Saúde, 2(4), 98. https://doi.org/10.51161/rems/2242

Massena, T. P., Lima, A. S. D., Soares, V. L., Ramos, I. S. M., Rezende, G. L. (2021). Aspectos da vulnerabilidade de adolescentes frente ao HIV. Revista Multidisciplinar Em Saúde, 2(4), 98. https://doi.org/10.51161/rems/2242

Osorio, R. G. (2021). A desigualdade racial no Brasil nas três últimas décadas. Instituto de Pesquisa Econômica Aplicada.- Brasília : Rio de Janeiro : Ipea. ISSN $1415-4765$ 
Research, Society and Development, v. 10, n. 16, e600101624045, 2021

(CC BY 4.0) | ISSN 2525-3409 | DOI: http://dx.doi.org/10.33448/rsd-v10i16.24045

Paiva, V. (2012). Cenas da vida cotidiana: metodologia para compreender e reduzir a vulnerabilidade na perspectiva dos direitos humanos. In: Vulnerabilidade e Direitos Humanos: Prevenção e promoção da saúde. Livro I - da doença à cidadania. Curitiba: Juruá Editora.

Panarra BACS, Teixeira E, Palmeira IP, Rodrigues ILA, Ferreira AMR. (2017). Vítimas e culpadas: representações sociais sobre mulheres que vivem com HIV. Rev Cuid.; 8(3): 1887-98. http://dx.doi.org/10.15649/cuidarte.v8i3.451

Papalia, D. E; Martorell, G. Desenvolvimento humano. 14. ed. Porto Alegre: AMGH, 2021.

Pessoa, M. F., Vaz, D. V., \& Botassio, D. C. (2021). Viés de gênero na escolha profissional no Brasil. Cadernos de Pesquisa, 51, e08400. http://200.155.188.131/index.php/cp/article/view/8400

Ratinaud, P. (2014). IRAMUTEQ: Interface de R pour les Analyses Multidimensionnelles de Textes et de Questionnaires - 0.7 alpha 2. http://www.iramuteq.org.

Santos, S. M. S. \& Oliveira, M. L. F. (2009). Conhecimento sobre AIDS e drogas entre estudantes de graduação de uma instituição de ensino superior no estado do Paraná. Revista Latino-Americana de Enfermagem. 17(4), 522-8. www.eerp.usp.br/rlae.

Silva S. M., Ferreira, M. M., Amaral-Bastos, M. M., Monteiro, M. A., Couto, G. R. (2020). Diagnóstico do conhecimento dos adolescentes sobre sexualidade. Acta Paul Enferm. eAPE20190210. DOI http://dx.doi.org/10.37689/acta-ape/2020AO0210

Silva, V. M., Amorim, R. de J. M., Barros, R. C. do R., Javorski, M., Queiroga, B. A. M. de, Andrade, R. T. de, \& Lima, L. S. de. (2018). Fatores Relevantes na Iniciação Sexual: Discurso Coletivo de Adolescentes em uma Escola do Recife, Brasil. Revista Brasileira de Sexualidade Humana, 29(1), 30-38. https://doi.org/10.35919/rbsh.v29i1.39

Wagner, T. M. C., Maggi, A., Souza, C. T. \& Souza, R. S. (2010). Estudantes Universitários em Tempos de HIV. Interação em Psicologia, $14(1), 61-71$.

Wohlgemuth, M. G. C. L, Polejack, L., \& Fleury Seidl, E. (2020). Jovens universitários e fatores de risco para infecção pelo HIV: uma revisão de literatura. RELACult - Revista Latino-Americana de Estudos em Cultura e Sociedade, 6(1). https://doi.org/10.23899/relacult.v6i1.1631

Wohlgemuth, M. G. C. L., Fatores de risco e proteção para infecção pelo HIV na perspectiva de estudantes universitários. 2020.154 f., il. Dissertação (Mestrado em Psicologia Clínica e Cultura)_Universidade de Brasília, Brasília, 2020.

Zabalza, M. A. (2004). O ensino universitário: seu cenário e seus protagonistas. Porto Alegre: Artmed. 